\title{
Activating photonic crystal membrane nanocavities by infiltrating with liquid crystals or luminescent colloidal nanocrystals
}

\author{
Mehmet A. Dündar ${ }^{* a}$, Christina Christova ${ }^{\mathrm{a}, \mathrm{b}}$, Andrei Yu. Silov ${ }^{\mathrm{a}}$, Fouad Karouta ${ }^{\mathrm{a}}$, Richard Nötzel ${ }^{\mathrm{a}}$, \\ Martijn Wienk ${ }^{\mathrm{b}}$, Huub Salemink ${ }^{\mathrm{c}}$, Rob W. van der Heijden ${ }^{\mathrm{a}}$ \\ ${ }^{a}$ COBRA Research Institute, Eindhoven University of Technology, PO Box 513, \\ NL 5600 MB Eindhoven, The Netherlands \\ ${ }^{\mathrm{b}}$ Department of Chemical Engineering and Chemistry, Eindhoven University of Technology, PO \\ Box 513, NL 5600 MB Eindhoven, The Netherlands \\ ${ }^{\mathrm{c}}$ Kavli Institute of Nanoscience, Delft University of Technology, P.O. Box 5053, \\ NL 2600 GB Delft, The Netherlands
}

\begin{abstract}
Liquid crystal (LC, Merk 5 CB) is infiltrated into active, InAs quantum dots embedded, InGaAsP membrane type nanocavities to investigate the possible effect of the LC orientation on active cavity tuning. The tuning is demonstrated thermally and thermo-optically. The thermal tuning showed that the cavity modes can be tuned in opposite directions and exhibits a sudden change at the clearing temperature. The mechanism relies on the existence of both ordinary and extraordinary refractive indices of the liquid crystal due to its molecular alignment inside the voids. It shows that the electric field distribution of cavity modes can have a substantial component parallel to the LC director. The average electric field orientation with respect to the LC orientation can be mode dependent, so that different modes can be dominated by either branch of the LCs refractive index. Thermo-optic tuning of the modes is obtained when the power of the excitation laser is increased from $40 \mu \mathrm{W}$ to $460 \mu \mathrm{W}$. A large and a reversible blueshift of more than $10 \mathrm{~nm}$ of the cavity modes is observed which is attributed to temperature induced liquid transport. InGaAsP type of nanocavities, without InAs quantum dots were infiltrated with PbSe colloidal quantum dots to obtain a comparison of internal light sources either in the semiconductor or in the holes.
\end{abstract}

Keywords: Photonic crystal, infiltration, tuning, liquid crystal, colloidal quantum dots

\section{INTRODUCTION}

Photonic crystal nanocavities have attracted much attention in both fundamental research and application areas of photonics because they can confine light in a small modal volume $(\mathrm{V})$ with a very high quality factor $(\mathrm{Q})$. The cavities having a high $\mathrm{Q} / \mathrm{V}$ ratio have been used in the investigation of cavity quantum electrodynamics [1]. These cavities have also found a wide application in opto-electronics as ultralow-threshold lasers [2], add-drop filters [3] and optical switches [4].

Both the fundamental and the application areas require large, fast and active tunability of the cavity modes. Therefore, tuning mechanisms have been demonstrated that rely on changing the effective refractive index via temperature [5] or by inserting scanning probe tips inside the air holes [6]. Another promising approach is to change the refractive index of the cavity environment by infiltrating liquids. Liquid crystal (LC) has been widely used as an infill because its refractive index varies by temperature or electric field. LC is also attractive because it has two different refractive indices, the ordinary and extraordinary refractive indices, in the nematic state. Infiltration techniques and the effects of the infiltrated LC have been widely studied, in particular for two-dimensional (2D) photonic crystal structures. In these studies, shifts of the band-gap edges and the lasing mode have been observed by adjusting either temperature or electric field [7-10].

Colloidal QDs have been used before as an internal light source to characterize passive structures $[12,13]$. The ability to dilute colloidal QDs makes them a promising candidate to obtain single QD interactions with the photonic states. So far, single photon generation at room temperature has been observed by using CdSe QDs; however these structures suffer

*m.dundar@tue.nl: phone 3140 2472976; fax 31402461339

Photonic Crystal Materials and Devices IX, edited by Hernán R. Míguez, Sergei G. Romanov, Lucio Claudio Andreani, Christian Seassal, Proc. of SPIE Vol. 7713, 77130E

(c) 2010 SPIE $\cdot$ CCC code: $0277-786$ X/10/\$18 $\cdot$ doi: 10.1117/12.854211

Proc. of SPIE Vol. 7713 77130E-1 
from the low emission efficiency [11]. PbS and PbSe colloidal QDs have been used to characterize passive devices by embedding the QDs in a polymer matrix [12]. The long lifetime and high luminescence efficiencies have been observed at the telecommunication wavelength in Si based cavities [13].

In this work, we demonstrate active tuning of liquid crystal infiltrated InGaAsP photonic crystal nanocavities incorporating InAs quantum dots by changing the temperature and applied laser power. Birefringence-induced mode dependent tuning of the cavity modes were observed by changing the temperature. We attribute the opposite tuning of the cavity modes to LC molecular orientation inside the holes which is most likely escaped radial configuration. Reversible blueshifts of the cavity modes by more than $10 \mathrm{~nm}$ were observed under laser irradiation. These are attributed to temperature induced LC transport in-and-out of the cavity as a result of laser-induced heating. We also infiltrated $\mathrm{PbSe}$ colloidal quantum dots into the passive InGaAsP cavities air holes, as an alternative to the InAs quantum dots inside the semiconductor. High photoluminescence enhancement and strong emission at the cavity modes were observed.

\section{FABRICATION OF THE CAVITIES AND EXPERIMENTAL SET-UP}

\subsection{Photonic crystal nanocavity fabrication}

To fabricate the photonic crystal membrane nanocavities, a $220 \mathrm{~nm}$ thick InGaAsP quaternary layer was grown on $1 \mu \mathrm{m}$ InP buffer layer by using Metal-Organic Chemical Vapor Deposition [14]. The active cavities have a layer of selfassembled InAs QDs with a density of $3 \times 10^{10} \mathrm{~cm}^{-2}$. A $20 \mathrm{~nm} \mathrm{InP}$ capping layer was grown on the top to protect the sample. Then, the sample is transferred to Plasma Enhanced Chemical Vapor Deposition to deposit a $400 \mathrm{~nm} \mathrm{SiN}_{\mathrm{x}}$ mask. A $350 \mathrm{~nm}$ thick positive electron beam resist ZEP 520 A was spin-coated after which the sample was postbaked at 200 ${ }^{\circ} \mathrm{C}$ for two minutes. The hexagonal array photonic crystal cavities were defined by $30 \mathrm{keV}$ Electron Beam Lithography with different lattice spacing and a constant $r / a$ ratio of 0.3 . The pattern is transferred to Reactive Ion Etching with $\mathrm{CHF}_{3}$ chemistry to open a $400 \mathrm{~nm} \mathrm{Si}{ }_{3} \mathrm{~N}_{4}$ mask. Then the pattern is created in the semiconductor layer stack by Inductively Coupled Plasma etching using $\mathrm{Cl}_{2}: \mathrm{Ar}_{2} \mathrm{H}_{2}$ chemistry. The final step was a wet chemical etching of the InP to release the InGaAsP membrane using $\mathrm{HCl}: \mathrm{H}_{2} \mathrm{O}=4: 1$ chemical mixture at $2{ }^{\circ} \mathrm{C}$. Figure 1 (a) and (b) show the Scanning Electron Microscope (SEM) images of the fabricated one (H1) and three (L3) missing air hole(s) cavities respectively. The six air holes around the $\mathrm{H} 1$ cavity and two air holes, in $\mathrm{\Gamma}-\mathrm{K}$ direction, of L3 cavity are modified by changing their size and/or position. The $\mathrm{H} 1$ and $\mathrm{L} 3$ cavities have the same lattice spacing of $480 \mathrm{~nm}$. The radius of the six surrounding holes of the $\mathrm{H} 1$ cavity is reduced by $33 \mathrm{~nm}$ and the center positions are shifted radially outwards by $13 \mathrm{~nm}$. The radius of two holes of the L3 cavity is kept the same but they are shifted laterally in opposite directions by $30 \mathrm{~nm}$. Due to the fabrication tolerances, the fabricated nanocavities have $r / a$ ratio of 0.32 instead of the intended 0.30 , as determined from the SEM images.
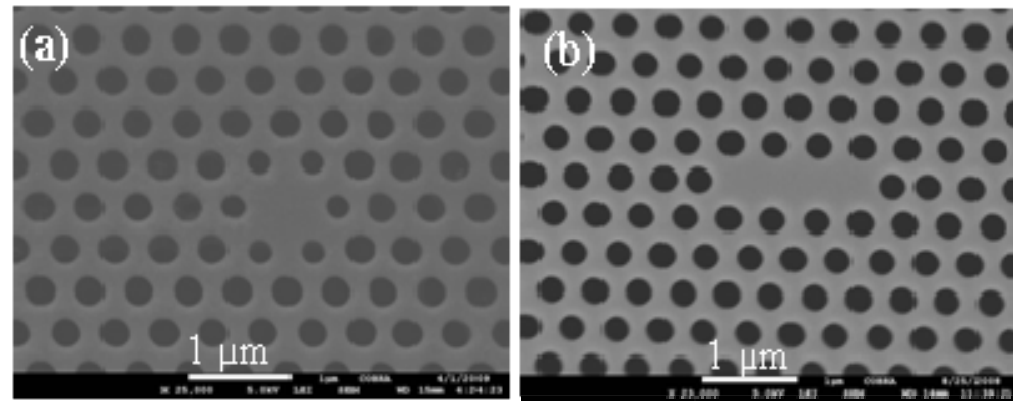

Figure 1. SEM images of (a) the H1 and (b) the L3 cavities.

The fabricated active and passive cavities are treated by $\mathrm{O}_{2}$ plasma and then they are rinsed in $\mathrm{H}_{3} \mathrm{PO}_{4}: \mathrm{H}_{2} \mathrm{O}$ solution to increase hydrophilicity of the surface in order to obtain a good wetting for the infiltrations. The contact angle is occasionally measured and less than $15^{\circ}$ is observed. In our experiments, the LC 4-pentyl-4'cyanobiphenyl (5CB, Merck), which has the nematic-isotropic phase transition temperature, or clearing temperature, $T_{\mathrm{c}}$ at $35^{\circ} \mathrm{C}$, is infiltrated into the cavities under ambient pressure. This is done by placing a small drop of the LC on top of the sample and removing the excess liquid by blowing dry $\mathrm{N}_{2}$. The PbSe colloidal QDs are infiltrated into passive cavities under oxygen and water-free atmosphere in a glovebox. A drop of $1 \mathrm{mg} / \mathrm{ml} \mathrm{PbSe}$ solution in chloroform was placed on the top of the sample. After evaporation of the chloroform, the sample is kept in a $\mathrm{N}_{2}$ atmosphere in a sealed chamber to avoid oxidization. 


\subsection{Experimental set-up}

Room temperature micro-photoluminescence (PL) measurements were conducted to investigate the nanocavities. The samples were mounted on an X-Y-Z stage, accommodated with a current controlled heating stage to vary the temperature for the thermal tuning. A CW He-Ne laser $(\lambda=632 \mathrm{~nm}$, for thermal tuning) or a power tunable $\mathrm{CW}$ diode laser $(\lambda=660$ $\mathrm{nm}$, for thermo-optic tuning) was focused through high numerical aperture microscope objective (100x, N.A $=0.55$ or 50x, N.A=0.45). Both laser spots have around $4 \mu \mathrm{m}$ spot diameters and the He-Ne laser power is $13 \mu \mathrm{W}$ on the samples. Excitation of the cavities and the collection of PL emission were done by the same objective. The collected PL was then dispersed in a monochromator and detected by liquid nitrogen cooled InGaAs array.

\section{THERMAL AND THERMO-OPTIC TUNING OF THE LC INFILTRATED CAVITIES}

\subsection{Thermal and thermo-optic tuning of empty cavities}

In order to investigate the thermal and thermo-optic effects on LC infiltrated cavities; we first conducted experiments by using unfilled active $\mathrm{PhC}$ nanocavities as a reference. We obtained the thermal tuning by varying the temperature of the sample using the heating stage at low power of the excitation laser. Figure 2(a) shows the temperature dependent wavelength tuning of the $\mathrm{H} 1$ cavity. The modes were identified by using 3-D Finite Difference Time Domain calculation (CrystalWave). As the temperature is increased from $20^{\circ} \mathrm{C}$ to $64{ }^{\circ} \mathrm{C}$, Fig. 2(a) shows that the modes, a split quadrupole mode (A and B), a hexapole mode (C), and a dipole mode (D), are almost linearly redshifted more than $6 \mathrm{~nm}$ at a rate of $0.1 \mathrm{~nm} / \mathrm{K}$. The tuning is caused by the change in the refractive index of the semiconductor by the temperature.

The thermo-optic tuning of a cavity is obtained by irradiating it with a focused laser and varying the power. Figure 2(b) shows three different PL spectra obtained from a modified H1 cavity as the power of the CW pump laser increases. The cavity has a lattice spacing of $511 \mathrm{~nm}$ and the six holes are reduced by $35 \mathrm{~nm}$ and shifted by $26 \mathrm{~nm}$ radially outward. The split quadrupole (A and $\mathrm{B}$ ) and hexapole (C) modes clearly redshift as the excitation power increases from $1 \mathrm{~mW}$ to 5 $\mathrm{mW}$. Significant decrease in both the Q values and the intensity is observed as the pump power increases as a consequence of the sample heating. The modes are redshifted more than $15 \mathrm{~nm}$ as the applied power is increased to 5 $\mathrm{mW}$. The redshift of the modes is attributed to the change in the refractive index of the cavity due to cavity temperature increase. The increase is estimated to be more than $150{ }^{\circ} \mathrm{C}$ by using the calibrated heating experiment. The large temperature increase explains the reduced cavity emission at the higher values, related to increased nonradiative recombination from the QD's. Both thermal and thermo-optic tuning are reversible and no hysteresis has been observed.
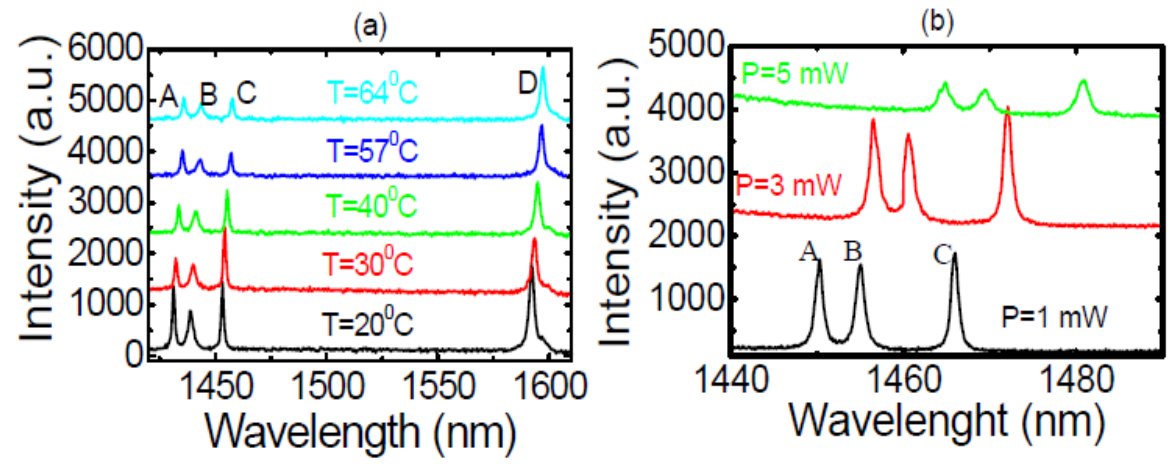

Figure 2. (a) Thermal and (b) thermo-optic tuning of two different empty H1 cavities.

\subsection{Thermal and thermo-optic tuning of empty cavities}

The LC is a birefringent material having two different refractive indices, ordinary and extraordinary, in the nematic state depending on the incident optical polarization. Figure 3(a) represents the temperature dependence of the ordinary $\left(n_{0}\right)$ and the extraordinary $\left(n_{\mathrm{e}}\right)$ refractive index of the LC $5 \mathrm{CB}$ for the wavelength of $1.5 \mu \mathrm{m}$, calculated from the parameters given in Ref. [15]. The $n_{\mathrm{o}}$ and the $n_{\mathrm{e}}$ have an opposite temperature dependence, which varies slightly with temperature below $T_{\mathrm{c}}$ and shows an abrupt change at $T_{\mathrm{c}}=35^{\circ} \mathrm{C}$, when both $n_{\mathrm{e}}$ and $n_{\mathrm{o}}$ become equal to the isotropic refractive index $n_{\mathrm{i}}$. The isotropic refractive index does not change with the temperature. Figure 3(b) shows the PL spectrum collected from the nanocavity, before infiltration. The modes are identified by using the 3-D FDTD calculation. The peaks occurring at $1461 \mathrm{~nm}$ and $1468 \mathrm{~nm}$ are quadrupole modes, referred to as the $\mathrm{Q}_{1}$-mode and the $\mathrm{Q}_{2}$-mode respectively. The 
$\mathrm{Q}_{1}$-mode and the $\mathrm{Q}_{2}$-mode are degenerate in ideal cavities, but in practice are split due to fabrication tolerances. The peak occurring at $1479 \mathrm{~nm}$ is the hexapole mode, referred to as the H-mode. Figure 3(c) shows the spectrum of the same cavity after the infiltration of the LC, which is done with the LC in the nematic state. All resonant modes are redshifted by more than $70 \mathrm{~nm}$ due to the change in the ambient refractive index. Since the filling is determined by wetting and governed by capillary forces inside the holes, the observed redshift after the infiltration is smaller than what we expected from the 3D FDTD calculations. Therefore, we simulated three different filling conditions: (1) total filling, (2) surface coverage and filling of the holes, and (3) surface coverage only, by using environment refractive index of 1.52. The simulation results suggest more than $100 \mathrm{~nm}$ redshift of the cavity modes after the infiltration in the case of total filling. Presumably, the undercut region is not filled. The simulation result is similar to the experimental result when we consider the second filling condition where surface is covered and holes are filled. Figure 3(d) shows the temperature dependent wavelength shift of the three modes. As the temperature is increased from $22^{\circ} \mathrm{C}$ to $44^{\circ} \mathrm{C}$, the $\mathrm{Q}_{1}$-mode and the H-mode redshift by more than $9 \mathrm{~nm}$ and $6 \mathrm{~nm}$ respectively, with an abrupt jump of $4 \mathrm{~nm}$ and $2 \mathrm{~nm}$ at the phase transition temperature. The redshift can be partly accounted for by the temperature dependence of the refractive index of the semiconductor, corresponding approximately to $0.1 \mathrm{~nm} / \mathrm{K}$; however, the $\mathrm{Q}_{2}$-mode blueshifts by more than $3 \mathrm{~nm}$, with a jump of around $1.5 \mathrm{~nm}$ at the transition point. The redshift and the blueshift are attributed to the increase in the ordinary and the decrease in the extraordinary branch of the LCs refractive index. It implies that the electric field distribution of $\mathrm{Q}_{2}$-mode has a substantial component parallel to the LC director. The average electric field orientation with respect to the LC orientation can be mode dependent, so that different modes can be dominated by either branch of the LCs refractive index [16].
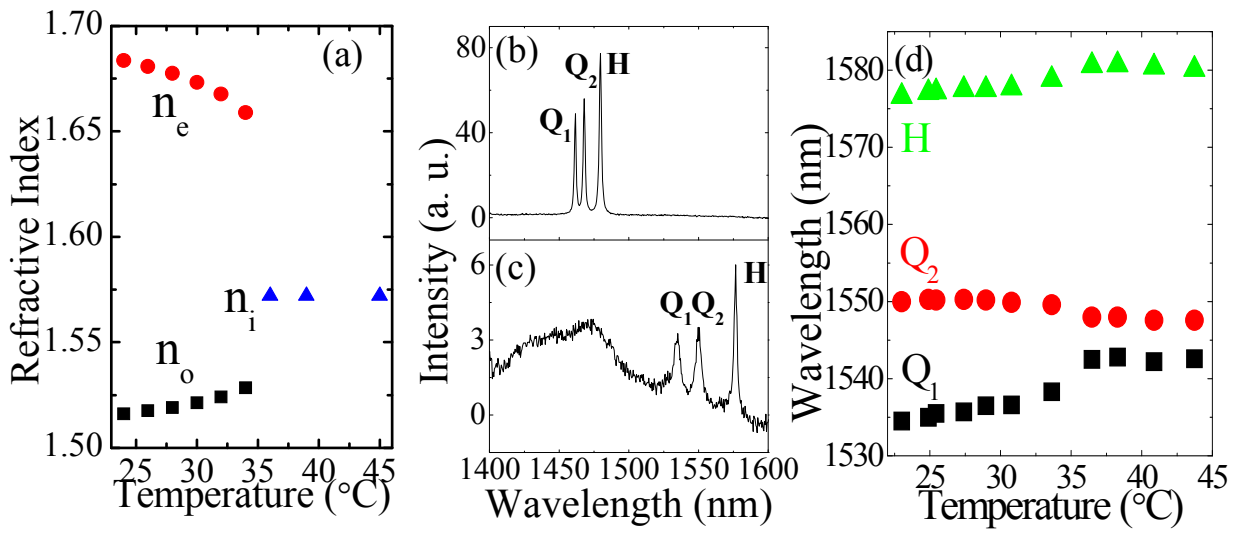

Figure 3: a) Temperature dependence refractive index change of the LC. b) PL spectrum of the nanocavity before the infiltration c) after the infiltration of the LC.d) The resonant wavelength shift of the modes with the increase of the temperature from $22{ }^{\circ} \mathrm{C}$ to $44{ }^{\circ} \mathrm{C}$.

The LC orientation is determined by sidewall anchoring, surface energy and molecular elasticity. Depending on these effects, three LC molecular orientations inside small diameter voids are theoretically proposed: uniform axial, planar polar, and escaped radial, as sketched in Figure 4 [17]. For the uniform axial orientation, Fig. 4(a), only $\mathrm{n}_{\mathrm{o}}$ would be relevant for the TE-polarized cavity modes. Indeed, several papers suggest this orientation inside photonic crystal holes, notably in large hole diameter $\mathrm{Si}$ [18] and in deeply etched (InGa)(AsP) [19]. In other works, evidence is presented for the escaped radial configuration (Fig. 4(b)), particularly well below $T_{c}[20]$ which has a significant amount of in-plane orientation. 


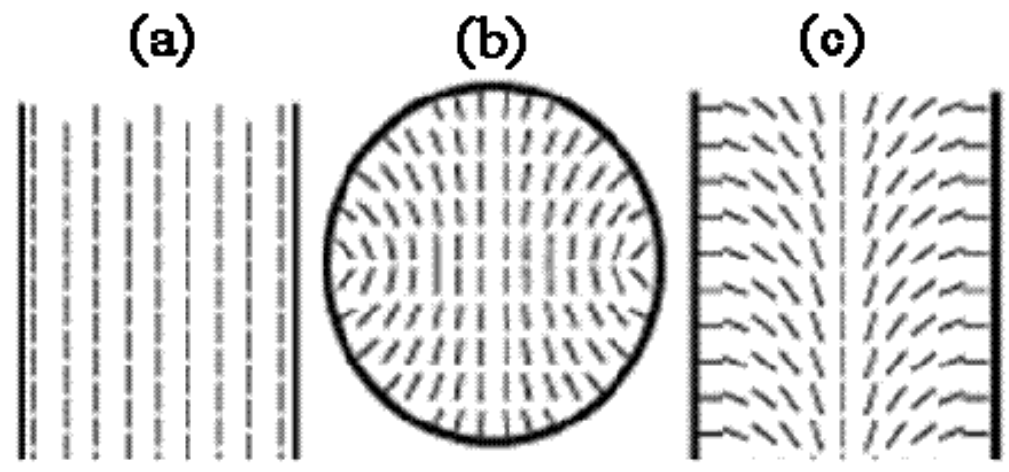

Figure 4: Schematic of a) axial uniform, b) planar, and c) escaped radial type of the LC alignment in the holes.

\subsection{Thermo-optic tuning of the $L C$ infiltrated cavities}

We investigated the tuning of LC infiltrated $\mathrm{PhC}$ nanocavities by thermo-optic effects. Figure 5(a) shows the mode tuning of another $\mathrm{H} 1$ cavity having the same lattice spacing of $480 \mathrm{~nm}$ and the surrounding holes are reduced by $34 \mathrm{~nm}$ and shifted by $24 \mathrm{~nm}$. As the applied laser power is increased from $40 \mu \mathrm{W}$ to $460 \mu \mathrm{W}$, all modes are blueshifted by more than $10 \mathrm{~nm}$ including an abrupt shift around $170 \mu \mathrm{W}$. On the other hand, the experiments on the thermo-optic tuning of unfilled cavities have demonstrated strong redshift of the cavity modes as the incident laser power increases. From Fig. 3(d), the $n_{e}$ only has a small contribution to the refractive index. Therefore, the large blueshift must be attributed to a different mechanism. The mechanism is likely related to the laser induced mass transport in- and out of the cavity, due to the temperature, or temperature gradient increase around the cavity. The changes in Fig. 5(a) are reversible. For depleting the cavities from liquid, evaporation is a possible mechanism. For the re-infiltration, however, as the power decreases, it must be assumed that the LC flows back into the cavity due to the wetting of the LC on the sample. Note also an abrupt change near $170 \mu \mathrm{W}$, which could correspond to the clearing temperature of the LC. Figure 5(b) and (c) show the microscope image of the cavity before and after the laser excitation. Figure 5(b) shows that as the laser power increases the LC disappears from the cavity.
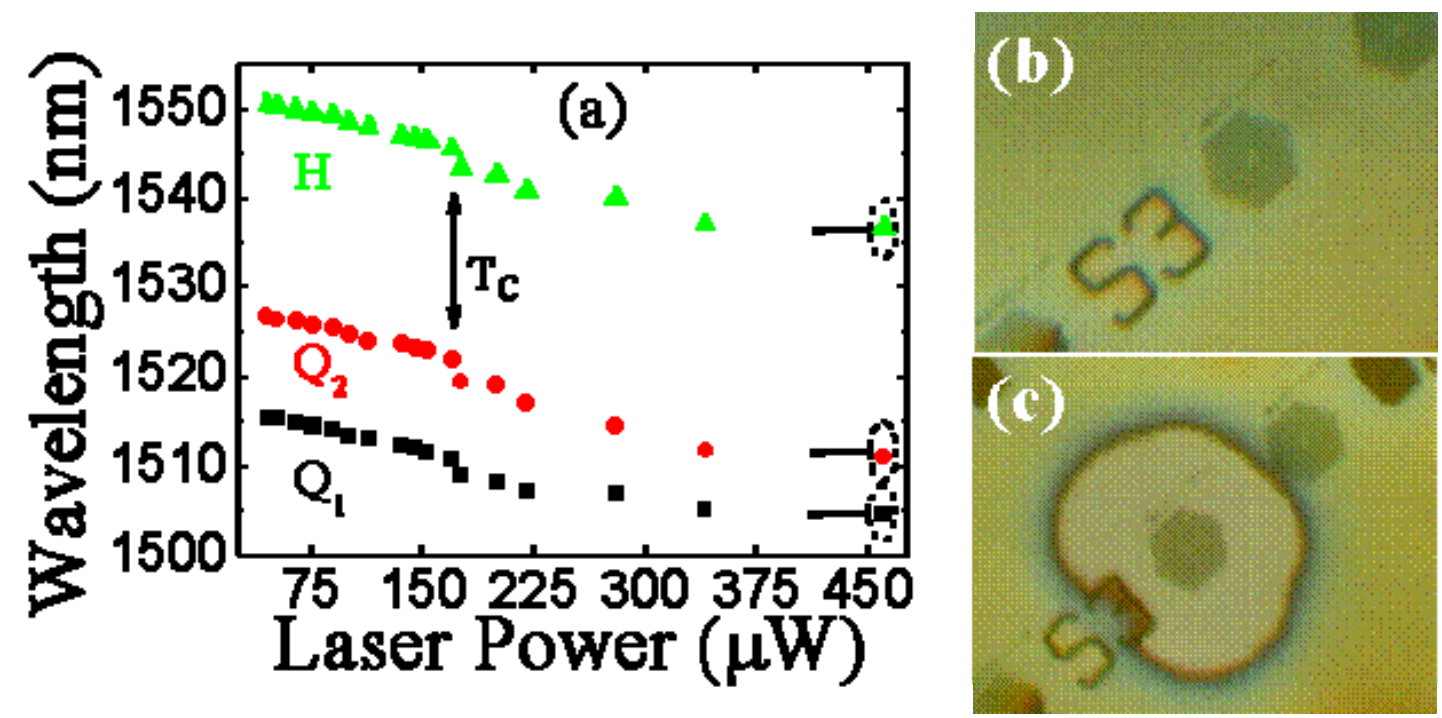

Figure 5. (a) Thermo-optic tuning of an H1 cavity, microscope picture of the H1 cavity (b) before and (c) after the LC transport. 


\section{COUPLING OF PBSE COLLOIDAL DOTS TO THE CAVITIES}

A high PL enhancement was observed from PbSe colloidal QDs under a weak CW excitation The QDs were deposited on top of an unprocessed InP sample and on the passive InGaAsP cavities, i.e. without InAs quantum dots. The samples were kept in an oxygen-free sealed chamber to avoid any photo-oxidization. Figure 6(a) shows the PL emission of the QDs from an unprocessed InP sample. The PL intensity has been constant for a long period of excitation. We also investigated the PL emission from the same InP sample under ambient medium with the same excitation power. Figure 6(b) and (c) are the PL emissions of the QDs collected from the same spot after 1 and 100 second(s) excitation. The PL intensity decreases due to photo-oxidization [21].
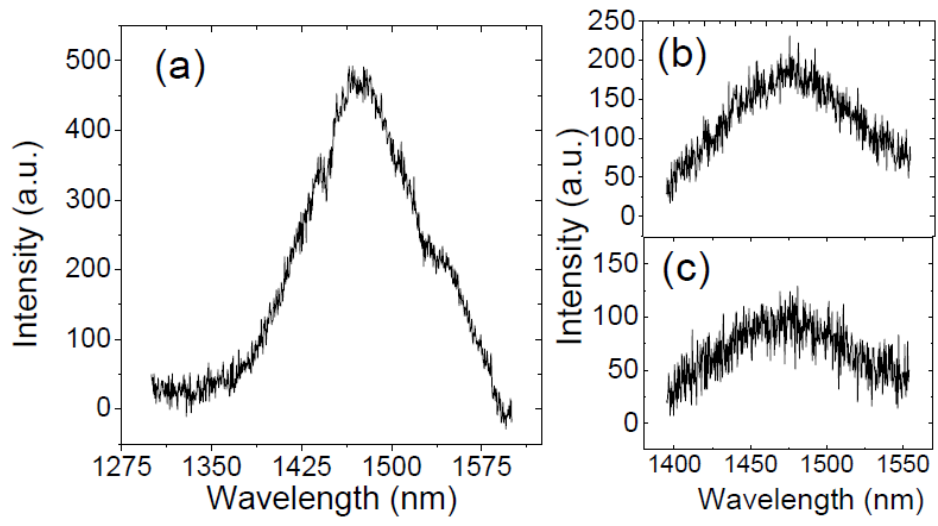

Figure 6. PL emission from the QDs (a) in oxygen-free medium, and in the ambient medium after (b) one and (c) one hundred second excitation time.

Figure 7(a) and (b) are collected from the H1 and the L3 cavities shown in Fig. 1(a) and (b)made from material without InAs quantum dots, after infiltration with the PbSe nanocrystals. The cavity modes are the same as for the active cavities with the same lithographic parameters. In Figure 7(a) the modes are split quadrupole (A and B) and hexapole (C) modes. They have Q factor more than 1000. The mode around $1375 \mathrm{~nm}$ in Fig. 1(b) is the fundamental mode having a Q factor of 600. The Q factors of both cavities are slightly smaller than the Q factors of the active cavities having the same lithographic parameters. The broad background in both figures is the QDs emission which is not coupled to the cavity mode.
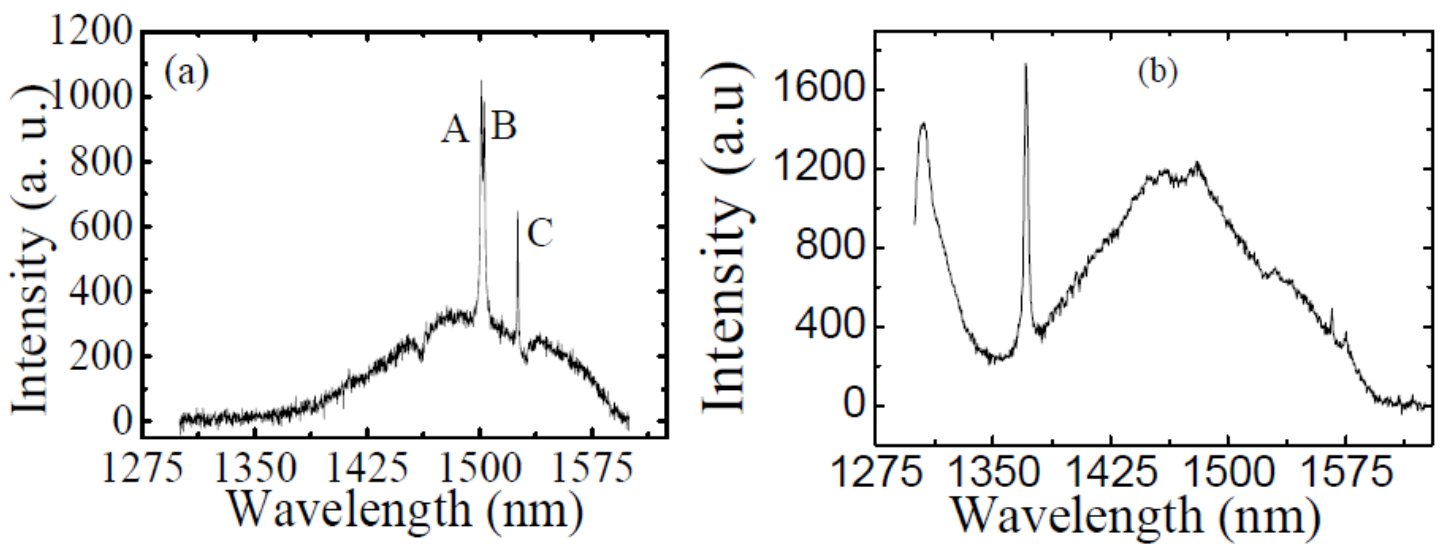

Figure 7. PL emission from (a) the H1 and (b) L3 cavities under oxygen-free medium. 


\section{CONCLUSION}

In this work, we investigated the activation of the passive and the active InGaAsP photonic crystal membrane type nanocavities by infiltration of liquid crystal or colloidal Quantum Dots. LC (5 CB, Merck) was infiltrated into active, InAs QDs embedded, InGaAsP membrane type of nanocavities. The resonant frequencies exhibited an abrupt change at the clearing temperature while different modes could be shifted in opposite directions. The latter is attributed to the mode dependent interactions with the ordinary and extraordinary refractive indices of the LC due to its molecular orientation distributions inside the holes. Large tuning effects were observed with varying laser power, which is tentatively attributed to temperature induced liquid transport. The epitaxial InAs QDs in the active membrane and the colloidal PbSe nanocrystals used in the passive membrane both had their emission wavelengths centered near $1.55 \mu \mathrm{m}$ and had comparable wavelengths spread. For the InAs case, the emission was entirely dominated by the cavity resonance, while for the PbSe case the resonances were strongly dominant above a broad background. The active and the passive cavities are promising candidates for the future optoelectronics devices.

\section{ACKNOWLEDGEMENTS}

The authors gratefully acknowledge useful discussions with C.W.M. Bastiaansen, D.J. Broer and A. Fiore, and thank P. A. M. Nouwens, B. Smalbrugge, E. J. Geluk, P. J. van Veldhoven, and T. de Vries for their help in the fabrication processes. Part of this research is supported by NanoNed, a technology programme of the Dutch Ministry of Economic Affairs via the foundation STW. This work is part of the research programme of the "Stichting voor Fundamenteel Onderzoek der Materie (FOM)", which is financially supported by the "Nederlandse Organisatie voor Wetenschappelijk Onderzoek (NWO)"

\section{REFERENCES}

[1] Kiraz, A., Reese, C., Gayral, B., Zhang, L. D., Schoenfeld, W. V., Gerardot, B. D., Petroff, P. M., Hu, E. L., and Imamoglu, A. "Cavity-Quantum Electrodynamics With Quantum Dots," J. Opt. B: Quantum Semiclassical Opt. 5, 129-137 (2003).

[2] Painter, O., Lee, R. K., Scherer, A., Yariv, A., O'Brien, J. D., Dapkus, P. D., and Kim, I. "Two-Dimensional Photonic Band-Gap Defect Mode Laser," Science 284, 1819-1821 (1999).

[3] Takano, H., Akahane, Y., Asano, T., and Noda, S. "In-Plane-Type Channel Drop Filter in a Two-Dimensional Photonic Crystal Slab," Appl. Phys. Lett. 84, 2226-2228 (2004).

[4] Takeda, H. and Yoshino, K. "Tunable Light Propagation in Y-Shaped Waveguides in Two-Dimensional Photonic Crystals Utilizing Liquid Crystals As Linear Defects," Phys. Rev. B 67, 073106 (2003).

[5] Faraon, A., Englund, D., Fushman, I., Vuckovic, J., Stoltz, N., and Petroff, P. "Local Quantum Dot Tuning on Photonic Crystal Chips," App. Phys. Lett. 90, 213110 (2007).

[6] Koenderink, A. F., Kafesaki, M., Buchler, B. C., and Sandoghdar, V. "Controlling the Resonance of a Photonic Crystal Microcavity by a Near-Field Probe," Phys. Rev. Lett. 95, 153904 (2005).

[7] Leonard, S. W., Mondia, J. P., van Driel, H. M., Toader, O., John, S., Busch, K., Birner, A., Gosele, U., and Lehmann, V. "Tunable Two-Dimensional Photonic Crystals Using Liquid-Crystal Infiltration," Phys. Rev. B 61, R2389-R2392 (2000).

[8] Schuller, C., Klopf, F., Reithmaier, J. P., Kamp, M., and Forchel, A. "Tunable Photonic Crystals Fabricated in III-V Semiconductor Slab Waveguides Using Infiltrated Liquid Crystals," App. Phys. Lett. 82, 2767-2769 (2003).

[9] Ferrini, R., Martz, J., Zuppiroli, L., Wild, B., Zabelin, V., Dunbar, L. A., Houdre, R., Mulot, M., and Anand, S. "Planar Photonic Crystals Infiltrated With Liquid Crystals: Optical Characterization of Molecule Orientation," Opt. Lett. 31, 1238-1240 (2006).

[10] Maune, B., Loncar, M., Witzens, J., Hochberg, M., Baehr-Jones, T., Psaltis, D., Scherer, A., and Qiu, Y. M. "Liquid-Crystal Electric Tuning of a Photonic Crystal Laser," Appl. Phys. Lett. 85, 360-362 (2004).

[11] Michler, P., Imamoglu, A., Mason, M. D., Carson, P. J., Strouse, G. F., and Buratto, S. K. "Quantum Correlation Among Photons From a Single Quantum Dot at Room Temperature," Nature 406, 968-970 (2000).

[12]Fushman, I., Englund, D., and Vuckovic, J. "Coupling of PbS Quantum Dots to Photonic Crystal Cavities at Room Temperature," Appl. Phys. Lett. 87, 241102 (2005). 
[13] Wu, Z., Mi, Z., Bhattacharya, P., Zhu, T., and Xu, J. "Enhanced Spontaneous Emission at $1.55 \mathrm{Mu}$ m From Colloidal PbSe Quantum Dots in a Si Photonic Crystal Microcavity," App. Phys. Lett. 90, 171105 (2007).

[14] Notzel, R., Anantathanasarn, S., van Veldhoven, R. P. J., van Otten, F. W. M., Eijkemans, T. J., Trampert, A., Satpati, B., Barbarin, Y., Bente, E. A. J. M., Oei, Y. S., de Vries, T., Geluk, E. J., Smalbrugge, B., Smit, M. K., and Wolter, J. H. "Self Assembled InAs/InP Quantum Dots for Telecom Applications in the $1.55 \mathrm{Mu} \mathrm{m}$ Wavelength Range: Wavelength Tuning, Stacking, Polarization Control, and Lasing," Jpn. J. Appl. Phys. 45, 6544-6549 (2006).

[15] Li, J. and Wu,S.T. "Extended Cauchy Equations for the Refractive Indices of Liquid Crystals," J. Appl. Phys. 95, 896-901 (2004).

[16] Dundar, M. A., Kicken, H. H. J. E., Silov, A. Y., Notzel, R., Karouta, F., Salemink, H. W. M., and Heijden, R. W. van der, "Birefringence-Induced Mode-Dependent Tuning of Liquid Crystal Infiltrated InGaAsP Photonic Crystal Nanocavities," Appl. Phys. Lett. 95, 181111 (2009).

[17] Burylov, S. V. "Equilibrium Configuration of a Nematic Liquid Crystal Confined to a Cylindrical Cavity," JETP 85, 873-886 (1997).

[18] Mertens, G., Roder, T., Matthias, H., Marsmann, H., Kitzerow, H. S. R., Schweizer, S. L., Jamois, C., Wehrspohn, R. B., and Neubert, M. "Two- and Three-Dimensional Photonic Crystals Made of Macroporous Silicon and Liquid Crystals," Appl. Phys. Lett. 83, 3036-3038 (2003).

[19] El-Kallassi, P., Ferrini, R., Zuppiroli, L., Le Thomas, N., Houdre, R., Berrier, A., Anand, S., and Talneau, A. "Optical Tuning of Planar Photonic Crystals Infiltrated With Organic Molecules," J. Opt. Soc. Am. B 24, 21652171 (2007).

[20] Schuller, C., Reithmaier, J. P., Zimmermann, J., Kamp, M., Forchel, A., and Anand, S. "Polarization-Dependent Optical Properties of Planar Photonic Crystals Infiltrated With Liquid Crystals," Appl. Phys. Lett. 87, 121105 (2005).

[21] Christova, C. G., Stouwdam, J. W., Eijkemans, T. J., Silov, A.Yu., Heijden, R.W. van der, Kemerink,M., Janssen, R. A. J., and Salemink, H. W. M. "Photoluminescence Enhancement in Thin Films of PbSe Nanocrystals," Appl. Phys. Lett. 93, 121906 (2008). 